\title{
Case series of feasibility considerations that impact operational delivery strategy in the highly competitive rheumatoid arthritis space in Asia
}

This article was published in the following Dove Press journal:

Open Access Journal of Clinical Trials

I February 2013

Number of times this article has been viewed

\author{
Karen Wai' \\ Lisa Marie Saldanha' \\ Elvira Zenaida Lansang' \\ Saumya Nayak' \\ Anish Sule ${ }^{2}$ \\ Ken J Lee ${ }^{3}$
}

'Feasibility and Site Identification Asia, Quintiles East Asia Pte Ltd, Singapore;

${ }^{2}$ Feasibility and Site Identification India, Quintiles India, Mumbai, India;

${ }^{3}$ Site Services Asia, Quintiles East Asia Pte Ltd, Singapore

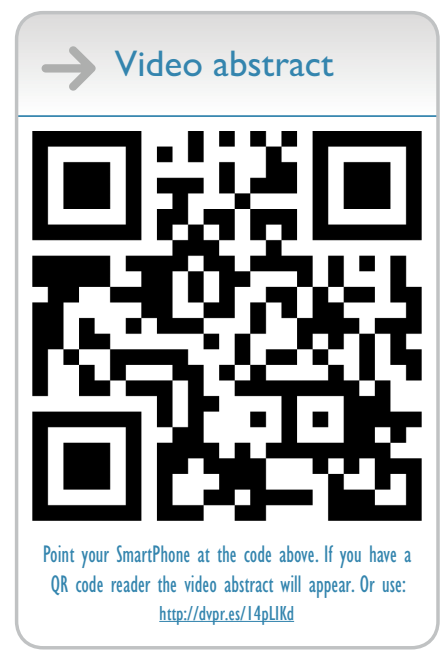

Correspondence: Karen Wai

Quintiles East Asia Pte Ltd, 79 Science

Park Drive, 06-08, Cintech IV, Science

Park One, Singapore 118264

Tel +656602 I 56 I

Fax +65 68720430

Email karen.wai@quintiles.com

\begin{abstract}
The rheumatoid arthritis (RA) clinical trial space is very competitive, and recruiting and retaining subjects is of critical importance. Feasibility studies are a central component of ensuring successful recruitment and retention. A feasibility study is an assessment of the practicality of a proposed study protocol, with the goal of understanding challenges and providing risk mitigation strategies leading to better subject enrolment and study start-up should the assessment be favorable. This paper presents findings from a retrospective case series of RA feasibilities, describing important parameters to consider in the highly competitive RA space in Asia. Key parameters identified and discussed are how decisions on clinical development strategy necessitate changes in the clinical operational delivery strategy, with focus on changes in inclusion and exclusion criteria and patient contribution load; how small the patient population becomes when the clinical trial needs to target the patient population that is refractory to standard therapy; regulatory timelines; and the competitive clinical trial landscape. Feasibility assessments are a snapshot in time exercise. Multiple parameters change over time, and, particularly in a space that has become competitive for subjects, one cannot rely on one static feasibility assessment to predict trial performance accurately. Continuous feasibility assessment will also provide insight into the resourcing needs on the part of the sponsor, contract research organization, and investigative site.
\end{abstract}

Keywords: site selection, country selection, clinical operations, risk management, recruitment rates

\section{Introduction}

Severe active rheumatoid arthritis (RA) is a chronic, systemic immune-mediated inflammatory disorder, affecting about $0.5 \%$ of the general population, in which the body attacks its own joints. This causes pain and swelling, and without appropriate treatment results in their progressive destruction. ${ }^{1-4}$ In addition, patients with RA suffer significantly increased cardiovascular morbidity and mortality, which is related not only to traditional cardiovascular risk factors but also to a chronic inflammatory state. ${ }^{5,6}$ Early treatment in RA is important because it can prevent disease progression and irreversible damage. ${ }^{4}$

Significant steps in the form of new therapies have been made in the management of more advanced disease, with three of the top 10 best-selling drugs worldwide in 2010 being novel monoclonal antibodies for RA or similar connective tissue disorders (infliximab, etanercept, and adalimumab), each having sales of around US\$7 billion. ${ }^{7}$ Despite this, there still remains unmet medical needs for additional therapies in RA, and biopharmaceutical drug development and therapy is therefore of considerable interest and importance in this therapeutic space..$^{2-4,8-13}$ This in turn means that the RA clinical trial space is very 
competitive, and recruiting and retaining subjects is of critical importance. Feasibility studies are a central component of ensuring successful recruitment and retention.

A feasibility study is an assessment of the practicality of a proposed study protocol, with the goal of understanding challenges and providing risk mitigation strategies leading to better subject enrolment and study start-up should the assessment be favorable. ${ }^{14}$ However, it is important to bear in mind that feasibility recommendations are based on an analysis at a specific point in time, and that factors impacting feasibility in the real world and those that affect the final feasibility analysis and recommendations are in a constant state of flux.

Between 2009 and 2011, our company received a higher than usual number of requests to conduct clinical trials in the RA space. Using this as a measurement of a highly competitive area, we analyzed our collated internal dataset in this therapeutic area and assessed a variety of feasibility parameters and their impact. This paper presents findings from a retrospective case series of RA feasibility analyses, describing important parameters to consider in the highly competitive RA space in Asia, with a focus on South Korea, Taiwan, and Malaysia.

\section{Case one}

A sponsor requested our company to perform a feasibility study on an international protocol in patients with RA who had an inadequate response to disease-modifying antirheumatic drugs (DMARDs) in late 2010. An investigator outreach was conducted using a questionnaire to assess protocol feasibility and obtain estimates on recruitment rates. Forty-eight sites/investigators were contacted to assess the target patient population that they would have access to, and to ascertain what percentage of that number would be suitable to be included in RA trials as determined by the inclusion and exclusion criteria presented to them. At this time point, the Asia feasibility team projected an estimated recruitment rate of 0.46 patients per site per month.

In early 2011, several significant changes took place within the program. Firstly, the sponsor was requested by the regulatory authorities to modify the patient population to those with disease refractory to tumor necrosis factor (TNF) inhibitors. As a consequence, there was a need to drop the projected average recruitment rate between December 2010 and March 2011, as well as increase the recruitment period. Alternatively, we would need to increase the number of sites in order to maintain the same recruitment period. Secondly, the sponsor made a strategic decision to exclude one region and allocate the clinical operations of those sites and patients to Asia.
This decision necessitated a two-fold increase in patient contribution to the study from investigative sites in Asia, requiring modifications to the recruitment period and/or number of sites. Another factor in the evolving landscape was the quantity of competitive clinical trials in the RA space. The feasibility group regularly scanned http://www.clinicaltrials.gov at intervals of 2-3 months, and monitored the type and number of clinical trials in the three main countries of interest for this protocol (South Korea, Taiwan, and Malaysia). The search term "rheumatoid arthritis" was used, and the results were filtered by "open studies" as well as the three countries of interest. For proprietary reasons, to illustrate the impact of the changes, we assumed that this study would recruit 100 patients across 10 sites at the initial assessment stage. All other calculations were extrapolated from this initial assumption. The impact on recruitment rate and period of these various changes and their respective time points is highlighted in Table 1.

\section{Case two}

Case two represents another study, this time straight off requiring patients failing TNF inhibitor therapy. This population of patients represents a potential strategic first target for developers of new RA compounds. However, they also represent a difficult population to recruit, as demonstrated by the survey of investigators conducted as part of the feasibility in the target countries for this protocol. In order to get to the TNF inhibitor failure population, one sees a strong funneling off effect from the total RA population. To illustrate these challenges, we describe here the results of the feasibilities conducted in Taiwan and South Korea. The investigators surveyed in South Korea treated a total of 1800 patients with RA in their practices over a 6-month period. Of these patients, only 80 were refractory to DMARDs and treated with TNF inhibitors. Within these 80 patients, only five individuals had an inadequate response to TNF inhibitors (as defined by insufficient efficacy or loss of efficacy after at least 3 months of treatment or intolerance of such treatment). Hence, as shown in Figure 1, the target population for this trial represents just $0.27 \%$ of the total RA population treated by the investigators surveyed. As shown in Figure 2, a similar pattern was seen in the Taiwan physician surveys, ie, a total of 700 RA patients seen over 6 months, with 52 being treated with TNF inhibitors and just three patients $(0.42 \%$ of the total population) being refractory.

\section{Case three}

Case three was a Phase II study of an immunosuppressive agent in patients with inadequate response to DMARDs (methotrexate). Phase II studies have a smaller sample size and are conducted 
Table I Major changes in sponsor strategy and consequent impact on recruitment rate and period

\begin{tabular}{|c|c|c|c|c|c|}
\hline Events & Date & Trials $^{\mathbf{a}}$ & $\mathbf{R R}$ & RP (months) & Sites $^{b}$ \\
\hline $\begin{array}{l}\text { Initial analysis and projection (major inclusion criteria } \\
\text { being failure of DMARDs) }\end{array}$ & December 2010 & 24 & 0.46 & 22 & 10 \\
\hline \multirow[t]{2}{*}{ Change in patient population to TNF inhibitor failures } & March 20II & 25 & 0.33 & 30 & 10 \\
\hline & & & & 22 & 14 \\
\hline Strategic decision to exclude a region from the clinical & June 2011 & 28 & 0.33 & 30 & 20 \\
\hline trial (increase in patients needed from Asia) & & & & 22 & 28 \\
\hline \multirow[t]{2}{*}{ Decision for start-up activities to begin } & August 20II & 29 & 0.33 & 30 & 20 \\
\hline & & & & 22 & 28 \\
\hline
\end{tabular}

Notes: aNumber of competing trials as per http://www.clinicaltrials.gov; recruitment period was calculated as the total number of patients divided by the number of sites divided by the recruitment rate (the method used here does not take into account the sigmoidal shape in terms of recruitment); bone investigator per site is assumed. Abbreviations: RR, recruitment rate; RP, recruitment period; DMARDS, disease-modifying antirheumatic drugs; TNF, tumor necrosis factor.

in a relatively smaller number of countries/sites that have shorter regulatory approval timelines with highly experienced investigators. When selecting the target Asian countries together with the sponsor, a number of factors were considered. Firstly, due to the toxicity profile of the investigational product, countries such as Indonesia and Philippines were eliminated due to a high prevalence of latent tuberculosis and risk of reactivation. China was not a possible candidate due to slow regulatory timelines that are not compatible with Phase II trials. Another consideration in preparing the proposal for this trial was that it was entering a more intensely competitive RA space than when the above described two feasibility assessments were requested. While the population with inadequate response to DMARDs was different from other trials we were running, overall site resources being consumed for research needed to be considered, and this was reflected in a more modest forecast in our proposal.

\section{Discussion Feasibility studies}

In 2011, our company was tasked to provide feasibility input for over 700 potential studies in Asia. Of these, 28 were in

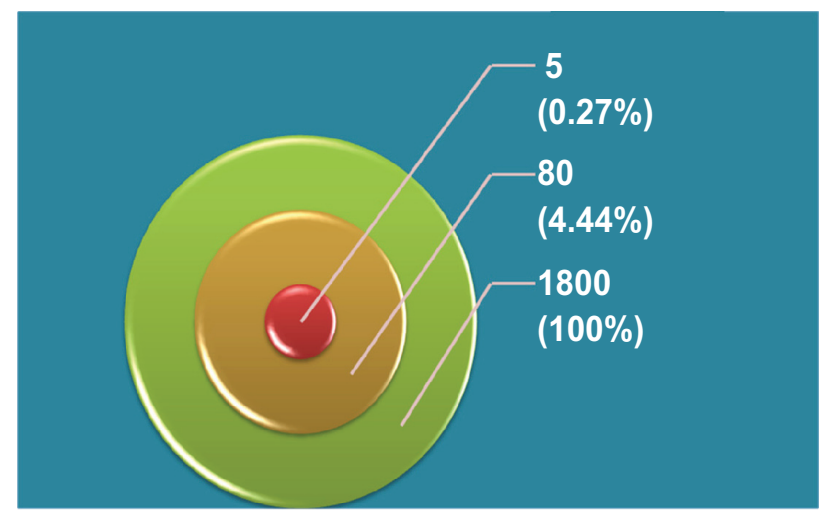

Figure I Patient funnel for South Korea showing a reduction in patient numbers at each treatment level. the field of RA, making this the fourth highest individual indication. In terms of distinct protocols, RA represented 4\% of the total conducted throughout Asia, with a total of 369 subjects recruited in 2011. In the first 6 months of 2012, 425 subjects were recruited, suggesting an increasing trend in terms of number of subjects recruited year on year. Given the large amount of activity in this space, a detailed and objective feasibility assessment is essential to ensure the success of a trial.

The logistics of operationally executing a clinical trial are enormous. ${ }^{14}$ Imagine a typical RA Phase III trial involving hundreds of subjects participating at a total of 80 investigational sites spread across several continents. Typically a sponsor will partner with a contract research organization (CRO) to design, execute, and analyze a clinical trial. Once a solid draft of the study protocol has been developed, the CRO's feasibility team is engaged. As Turner ${ }^{14}$ observed, "This team's role is to assess the full operational demands of the clinical trial, and to ask (and eventually answer) this question: can the trial be executed as currently laid out in the protocol?" To answer this question, the team initiates a series of internal and external investigative processes that will provide the necessary information.

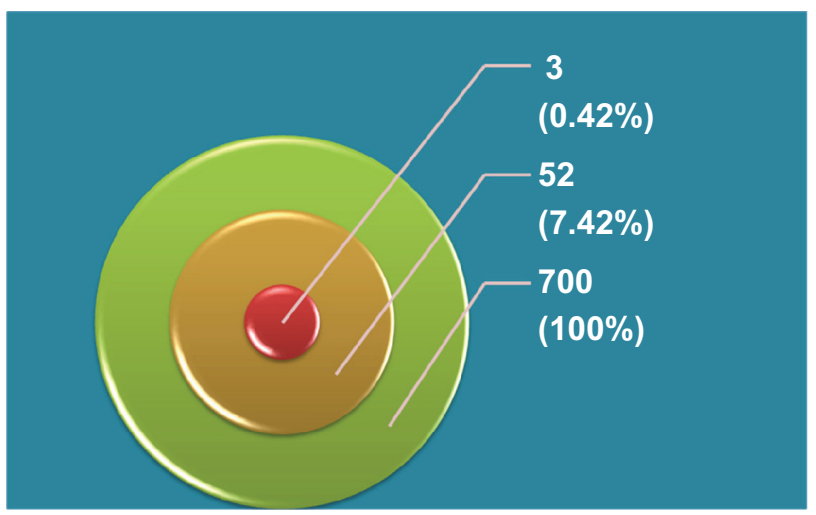

Figure 2 Patient funnel for Taiwan showing a reduction in patient numbers at each treatment level. 
One informative internal approach to evaluating the feasibility of a protocol is to check past performance metrics for other trials that the CRO has successfully completed in the same therapeutic area. Questions of interest include:

- Where were the sites used in the previous trials located?

- How easy was it to recruit and retain the required number of subjects for the previous trials, and did the ease of recruitment vary across geographic locations within countries and across countries?

It is also important to consider how similar the suggested study design for this particular trial is to the designs used for previous trials, with particular attention being paid to the number of subjects required.

After a relatively short period of time (perhaps less than one week) the feasibility team will typically make one of two decisions. The first is that they have an acceptably high degree of confidence that the study can be successfully executed as currently described in the protocol. The second possible decision is that initial research has not provided sufficient evidence to allow a good decision to be made. In this case, a more extensive evaluation process is implemented, and this can take in the order of 6-8 weeks. In conjunction with the CRO's medical advisors and project managers, the feasibility team creates a survey that is sent with the study synopsis or protocol to physicians in various locations at which the trial may be conducted. The survey asks a series of questions targeted at determining if the physicians would be able to recruit subjects into the trial and the timeline by which they would be able to do so. Our organization also conducts face-to-face interviews and focus groups depending on sponsor requests and protocol complexity. The benefits of these strategies include a better understanding of the proposal and more accurate analysis of the patient population. In parallel with the survey execution phase, feasibility team members will continue to mine data from both publicly and privately available databases to investigate the prevalence of the disease or clinical condition of concern, and to gain a solid understanding of the target patient population that is the focus of the study. These can include prescription and medical claims databases. Patient advocacy groups for some diseases can also be good resources of information. At this point in time, it is very important to consider the protocol's inclusion and exclusion criteria and to estimate how many individuals from the overall pool of individuals with the disease of interest would be eligible for enrolment in this particular study. The goal here is to obtain a realistic estimate of subject availability, ie, to decide if the number of subjects stated in the study protocol can reasonably be expected to be recruited and retained.

Another important aspect in the evaluative process is to estimate how many other sponsors are currently planning and/or conducting similar trials, and hence competing for the same patients. One strategy here is to access the web site http://www.clinicaltrials.gov, where sponsors are required to register their trial. The CRO may also get information directly from the sponsor who wishes to conduct this specific trial, given that many biopharmaceutical companies have "competitive intelligence" divisions that gather information on other companies' drug pipelines and upcoming trials. Other sources of information include commercially available databases, feedback from internal experts within our company, and discussions with strategic sites.

When the surveys mentioned previously are returned, the feasibility team carefully considers the feedback they provide. Physicians interested in participating in the trial as principal investigators often inflate (unconsciously or consciously) the number of subjects they say they can recruit. ${ }^{14}$ They also tend to make positive statements about the suitability of their facilities and their abilities to execute any particularly complicated aspects of the protocol. As Turner ${ }^{14}$ observed, "While such rose-tinted self-appraisals may initially make the physician's site look attractive for inclusion in the trial, subsequent site underperformance has a cascade of unfortunate consequences. Overall subject recruitment is negatively impacted, the sponsor's clinical development program is delayed, and, should the drug eventually be approved for marketing, patients who are prescribed the drug could have benefited from it earlier".

Upon completion of their assessment and analyses, the feasibility team assimilates the results into a comprehensive report that includes recommendations as to where the study should be conducted, how quickly subjects can be enrolled, considerations addressing any potential risks to meeting the enrolment timeline, and risk mitigation strategies that could be employed if necessary. This report is delivered to the sponsor, and representatives from the sponsor and the CRO can then discuss these results and modify the draft protocol as appropriate to increase the likelihood of the trial's successful and timely execution. The "due diligence role" of feasibility teams is therefore a critically important one.

\section{Standard of care for RA in Asia}

The standard of care for patients with RA is fairly uniform across Asia, with some variations in more advanced disease that depend on market access to new therapies, as outlined in Figure 3. 


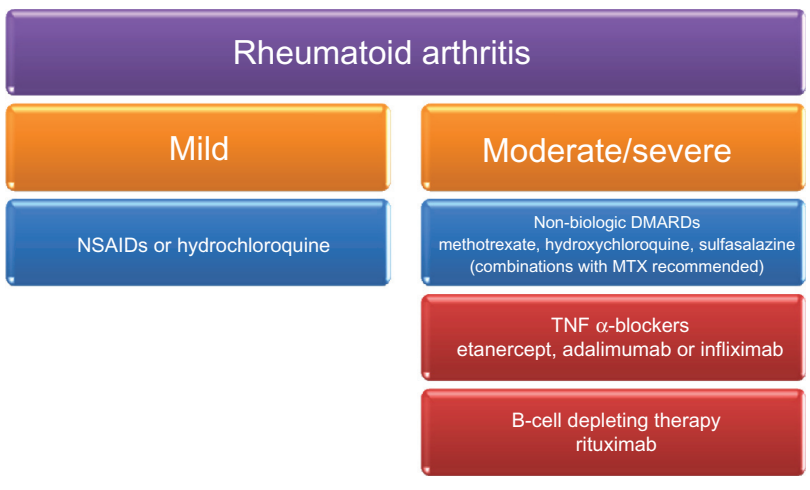

Figure 3 Treatment approaches for rheumatoid arthritis.

Abbreviations: DMARDS, disease-modifying antirheumatic drugs; NSAIDs, nonsteroidal anti-inflammatory drugs; MTX, methotrexate; TNF, tumor necrosis factor.

\section{Summary of information generated from investigator surveys in Asia}

Patients with confirmed moderate to severe RA typically start with methotrexate as monotherapy and progress if not sufficiently controlled to combination therapy with hydroxychloroquine or sulfasalazine. After 6 months, inadequate responders to methotrexate combination therapy typically progress to TNF inhibitors (infliximab, etanercept, and adalimumab). A new development in the treatment of RA is the B-cell depleting therapies (eg, rituximab) which only become a treatment option after failure of all other therapies.

Because these modern therapies are often expensive, their use is limited by patient affordability and reimbursement status. In South Korea, TNF inhibitors (infliximab, etanercept, and adalimumab) are provided by national health insurance using the copayment scheme where the patients have to pay $20 \%-40 \%$ of the cost of the treatment. In South Korea, nonbiologic DMARD treatment costs around US\$5 per dose (once a week) and TNF inhibitor treatment costs US \$110-520 per dose (every 2-8 weeks): hence, fewer patients opt for TNF inhibitor treatment. TNF inhibitors are fully reimbursed in Taiwan, and in Malaysia, there is limited reimbursements for RA patients in government hospitals treated by specialists.

\section{Operational strategy}

Several operational strategy considerations are evident from the cases. Firstly, sponsors are in constant consultation with the regulatory authorities on development strategies. Recruitment rates provide a basis to compute the recruitment period and the number of sites needed to provide the final number of patients to be enrolled in the protocol. Any decision on clinical development strategy (eg, patient population, inclusion and exclusion criteria) necessitates changes in the clinical operational delivery strategy (eg, number of sites, investigators, recruitment period). Increasing the number of sites/investigators at any point after initial assessment would most likely involve the addition of less experienced sites and investigators. There will always be a balance between operational risk and delivery. In addition to this risk, the sponsor's or CRO's operational team in the relevant countries would also have to be scaled up to manage the additional sites and patients, an action which also carries its own challenges.

Secondly, it is clear that if one were to start a clinical trial at a time when there are many other active trials in progress, one will need to ensure that there is a sufficiently large pool of patients available at the site and that these sites have the capacity to take on the additional study. In this respect, maintaining excellent relationships with sites is of key importance, because visibility of ongoing activities there will help with patient recruitment and risk mitigation strategies.

Thirdly, the importance of conducting an outreach to investigators to obtain a clear picture of the real world clinical situation can influence the choice of country.

The fourth point tackles the drug reimbursement status in a country. For RA, when new agents need to enter the refractory space, the target country must have sufficient use of TNF inhibitor drugs. Table 2 summarizes the reimbursement/ access situation throughout Asia for TNF inhibitors. Clearly, in countries without any reimbursement for TNF inhibitors, recruiting such patients can be a challenge. However, there can also be reasonable numbers of self-paying patients in these countries, and this important information needs to be teased out in the feasibility exercise to maximize the delivery of the trial.

The fifth point examines the issue of comorbidities. Potential latent infections, eg, tuberculosis and even high prevalence of other infectious diseases, eg, hepatitis B, pose a challenge for the newer immunosuppressive agents in development. Finally slow start-up timelines, as in China, provide another operational delivery issue that needs to be taken into consideration.

Table 2 Asian countries that can conduct a study in rheumatoid arthritis recruiting patients who are inadequate responders to at least one anti-tumor necrosis factor agent

\begin{tabular}{ll}
\hline Yes & South Korea, Thailand, Malaysia, Hong Kong, Australia, \\
& New Zealand \\
No & Singapore, Thailand, India, Philippines, Vietnam, China, \\
Indonesia, Sri Lanka
\end{tabular}

Note: Data from Quintiles internal proprietary data. ${ }^{5}$ 


\section{Conclusion}

Feasibility assessments are a snapshot in time exercise. Multiple parameters change over time, and particularly in a space that has become competitive for subjects, one cannot rely on one static feasibility assessment to predict trial performance accurately. Continuous feasibility assessment will also provide insight into the resourcing needs with regard to the sponsor, CRO, and investigative site. Knowledge of the magnitude of deliverables will enable project teams to manage risks proactively and ensure a sufficient lag time for scaling-up of resources. It is also prudent to investigate the external environment (eg, investigative sites, standard of care, and reimbursement status) to ascertain the available patient pool. This is especially important when the protocol targets patients who are refractory to standard therapy. In addition to the recruitment rates, recruitment period, and patient numbers, one must also be conscious of other mitigating factors that can impact the feasibility of a protocol, eg, regulatory start-up timelines. We will continue to monitor closely the performance of the trials presented here, and present an update of further "lessons learned" as new data become available.

\section{Acknowledgment}

The authors thank J Rick Turner for providing editorial assistance in the preparation of this paper.

\section{Disclosure}

The authors are employees of Quintiles, a provider of clinical trial services for biopharmaceutical companies worldwide. With regard to this specific paper, they do not have any competing interests.

\section{References}

1. PubMed Health. AHFS Consumer Medication Information, methotrexate. Available from: http://www.ncbi.nlm.nih.gov/ pubmedhealth/PMH0000547/. Accessed July 21, 2012.

2. Moura RA, Graca L, Fonseca JE. To B or not to B the conductor of the rheumatoid arthritis orchestra. Clin Rev Allergy Immunol. 2012;43: 281-291.

3. Nakken B, Munthe LA, Konttinen YT, et al. B-cells and their targeting in rheumatoid arthritis - current concepts and future perspectives. Autoimmun Rev. 2011;11:28-34.

4. Trouw LA, Mahler M. Closing the serological gap: promising novel biomarkers for the early diagnosis of rheumatoid arthritis. Autoimmun Rev. 2012;12:318-322.

5. Charles-Schoeman C. Cardiovascular disease and rheumatoid arthritis: an update. Curr Rheumatol Rep. 2012;14:455-462.

6. Amezaga Urruela M, Suarez-Almazor ME. Lipid paradox in rheumatoid arthritis: changes with rheumatoid arthritis therapies. Curr Rheumatol Rep. 2012;14:428-437.

7. Reuters Edition US. FACTBOX - World's top-selling drugs in 2014 vs 2010. Available from: http://www.reuters.com/article/2010/04/13/ roche-avastin-drugs-idUSLDE63C0BC20100413. Accessed July 21, 2012.

8. Furst DE, Keystone EC, Braun J, et al. Updated consensus statement on biological agents for the treatment of rheumatic diseases. 2011. Ann Rheum Dis. 2012;71 Supp1 2:2-45.

9. Levesque MC. Biologic rheumatoid arthritis therapies: do we need more comparative effectiveness data? Bio Drugs. 2012;26:65-70.

10. Nowell M, Evans L, Williams A. PBEF/NAMPT/visfatin: a promising drug target for treating rheumatoid arthritis? Future Med Chem. 2012;4: 751-769.

11. Demin I, Hamrén B, Luttringer O, Pillai G, Jung T. Longitudinal model-based meta-analysis in rheumatoid arthritis: an application toward model-based drug development. Clin Pharm Ther. 201;92:352-359.

12. Robak T, Robak E. Tyrosine kinase inhibitors as potential drugs for B-cell lymphoid malignancies and autoimmune disorders. Expert Opin Investig Drugs. 2012;21:921-947.

13. Vaddi K, Luchi M. JAK inhibition for the treatment of rheumatoid arthritis: a new era in oral DMARD therapy. Expert Opin Investig Drugs. 2012;21:961-973.

14. Turner JR. New Drug Development: An Introduction to Clinical Trials, 2nd ed. New York, NY: Springer; 2010.
Open Access Journal of Clinical Trials

\section{Publish your work in this journal}

The Open Access Journal of Clinical Trials is an international, peerreviewed, open access journal publishing original research, reports, editorials, reviews and commentaries on all aspects of clinical trial design, management, legal, ethical and regulatory issues, case record form design, data collection, quality assurance and data auditing

\section{Dovepress}

methodologies. The manuscript management system is completely online and includes a very quick and fair peer-review system, which is all easy to use. Visit http://www.dovepress.com/testimonials.php to read real quotes from published authors. 